\title{
LA MISE EN MOUVEMENT DANS LA PENSÉE MAGIQUE
}

Chantal Lheureux-Davidse

\section{ERES | «Cliniques méditerranéennes »}

$2012 / 1 n^{\circ} 85 \mid$ pages 7 à 22

ISSN 0762-7491

ISBN 9782749231891

Article disponible en ligne à l'adresse :

http://www.cairn.info/revue-cliniques-mediterraneennes-2012-1-page-7.htm

\section{Pour citer cet article :}

Chantal Lheureux-Davidse, "La mise en mouvement dans la pensée magique », Cliniques méditerranéennes 2012/1 ( $\mathrm{n}^{\circ}$ 85), p. 7-22.

DOI 10.3917/cm.085.0007

Distribution électronique Cairn.info pour ERES.

(C) ERES. Tous droits réservés pour tous pays.

La reproduction ou représentation de cet article, notamment par photocopie, n'est autorisée que dans les limites des conditions générales d'utilisation du site ou, le cas échéant, des conditions générales de la licence souscrite par votre établissement. Toute autre reproduction ou représentation, en tout ou partie, sous quelque forme et de quelque manière que ce soit, est interdite sauf accord préalable et écrit de l'éditeur, en dehors des cas prévus par la législation en vigueur en France. Il est précisé que son stockage dans une base de données est également interdit. 


\section{Chantal Lheureux-Davidse}

\section{La mise en mouvement dans la pensée magique}

Comment rendre vivant l'inanimé ? La pensée magique nous y aideraitelle ? L'entreprise reste difficile autant lorsqu'il s'agit de réanimer une relation peu vivante que lorsqu'il s'agit d'animer à distance des objets inertes. À travers le suivi de Flora, fillette autiste qui préférait l'environnement non humain aux liens humains en direct, la place de la pensée magique sera interrogée dans sa fonction structurante quant à l'investissement de l'espace environnemental et relationnel. Quel lien le thérapeute peut-il établir avec un enfant autiste quand celui-ci privilégie une pensée magique qui viserait à mettre en mouvement à distance des objets inanimés alors que le thérapeute paraît être considéré comme un objet inerte ? Je mettrai en valeur l'intérêt de jouer avec cette pensée magique pour que l'enfant se réapproprie spontanément le lien en direct.

\section{L'IMPOSSIBLE RENCONTRE EN DIRECT}

Flora, qui a renoncé très tôt à l'investissement du regard en direct, autisme oblige, a déplacé son intérêt pour la relation dans l'environnement non humain, c'est-à-dire dans celui des objets, du monde végétal et de l'architecture. Elle évite le regard en direct comme s'il était normal qu'elle se soit toujours débrouillée seule sans rien partager. Elle est pourtant très attentive à son environnement, pourvu qu'il ne soit pas humain.

Je la reçois chaque semaine dans le cadre d'une institution spécialisée, un $\mathrm{IME}^{1}$, en psychothérapie individuelle. Son retrait autistique rend encore les échanges trop imprévisibles pour elle lorsqu'ils sont en direct. Mais nous

Chantal Lheureux-Davidse, psychologue clinicienne, psychanalyste, maître de conférences à l'université Paris 7 ; 59, rue du Temple, F-75004 Paris - chantal.Iheureuxdavidse@laposte.net

1. Institut médico-éducatif. 
arrivons à partager l'intérêt pour les objets et les formes environnants, dans une coprésence qui peut nous convenir dans un premier temps.

Dès les premières séances, Flora supporte difficilement le contact. Elle ne sait pas se diriger seule dans l'espace et il est nécessaire de lui donner la main pour la guider d'un endroit à un autre. Mais lorsque je lui propose de me donner la main le temps des trajets, je constate qu'elle a une répulsion pour le contact ${ }^{2}$. Elle évite le contact en direct en cachant sa main dans sa manche. Je me demande si le langage que je lui adresse est compris dans un sens littéral comme il l'est par de nombreux enfants autistes. Craindrait-elle que je lui ôte la main si elle accepte de me « donner » la main, tant que son image du corps est encore mal construite ? Je lui propose alors de « se tenir à » ma manche faute de pouvoir me « donner » la main. Elle saisit immédiatement cette proposition ainsi que ma manche à laquelle elle s'agrippe, le temps de quelques trajets pour qu'elle s'habitue à ma présence et au fait que je ne risque pas de lui arracher une partie de sa main ni de la faire disparaître à mon contact. Au bout de quelques séances, elle fait l'essai fructueux et spontané pendant les trajets de me donner directement la main avec confiance. Dès lors, l'expérience du contact en direct n'est plus suivie d'un rejet, ni accompagnée d'émotions contradictoires.

Pour des personnes autistes la rencontre en direct est souvent très difficile au point que le contact peut entraîner le sentiment de disparaître ou de faire disparaître la partie du corps en contact.

\section{DISPERSION ET PENSÉE ASSOCIATIVE}

Sur le trajet vers mon bureau, Flora fait toujours le détour par une flaque d'eau. Elle teste le fond de la flaque en mettant son pied dans son reflet comme pour éprouver la solidité de ma capacité contenante avant chaque séance.

Quand je la raccompagne vers son groupe, elle s'arrête souvent pendant le trajet devant un magnifique massif de fleurs. Devant ce buisson fleuri au printemps, taillé dans une forme convexe et arrondie, elle regarde avec émerveillement tantôt les fleurs et tantôt mes yeux en énonçant avec exclamation de façon associative tout le registre de mots à son actif qui concerne les fleurs : « abeille, tartine, miel », mais sans pouvoir prononcer le mot « fleur». Tout le contexte y est mais sans le mot qui désigne ce qu'elle regarde. Entre chaque mot elle suscite mon approbation comme un petit pourrait attendre confirmation de l'intérêt de ses découvertes en sollicitant l'adulte du regard. Elle tourne alors la tête très lentement dans ma direction après avoir regardé

2. Cela m'évoque le témoignage de Donna Williams, jeune femme autiste, dans son livre : Si on me touche, je n'existe plus. 
les fleurs et exprimé quelques mots. J'ai l'impression que ses mouvements sont au ralenti et que cela lui permet d'être très présente. Elle partage ainsi ses émotions avec moi et les mots qu'elle exprime sont issus d'une pensée associative. Ainsi elle peut articuler l'éprouvé interne de ses émotions esthétiques à ce qu'elle voit et dans un langage partagé.

Les enfants autistes sont davantage dans une pensée associative, qui risque de dériver sans devenir pour autant délirante, que dans une pensée logique et contextualisée. C'est pourtant la pensée logique qui implique une présence à soi-même et aux autres. Ici l'environnement associatif du mot est préalable à la nomination du mot « fleur » qui désigne ce qu'elle voit.

\section{LA TERREUR DES FORMES INFORMES}

Lorsque Flora est dans mon bureau, elle a la terreur des rideaux et des cheveux qui ne sont pas des valeurs sûres et solides quant à la stabilité des leurs formes. Il lui arrive de lancer des objets en direction de ces formes informes vécues comme menaçantes et qui risqueraient de l'absorber ou de l'anéantir. Il lui faut des fonds solides et prévisibles dans son environnement pour se sentir en confiance. Elle cherche à éprouver les bords de l'espace, faute d'avoir pu intégrer la fonction contenante de la relation à l'autre. Elle est rassurée par une certaine constance de l'environnement, tant dans les formes solides de l'environnement non humain que dans l'authenticité des émotions exprimées du monde humain. Sans doute le manque d'accordage dans la relation première n'a pas permis à Flora de se sentir contenue.

Son handicap génétique, une trisomie 21 qui se voyait dès la naissance, avait sidéré la maman au point que l'accordage des regards avait été difficile, trop vite ambivalent et donc imprévisible pour le bébé. De plus, un papa démissionnaire qui ne se sentait pas capable d'occuper son rôle de père avait laissé la mère comme seule responsable de la situation. Ce contexte familial n'avait pas offert à Flora la chance de se sentir dans une place de choix. Pour l'entourage, elle était plutôt un bébé encombrant qui ne s'accordait pas dans la relation. Elle avait rendu la maman déprimée dès les premières années. La mère " fonctionnait » au mieux malgré tout, en faux-self, en tâchant d'apporter le meilleur mais sans conviction et sans offrir le luxe ni le plaisir de jouer avec son bébé et sans non plus le raconter. Cela ne favorisait pas l'installation des échanges relationnels et émotionnels. Ce bébé vivait dans un certain confort quant aux soins, mais ne pouvait s'ajuster ni dans sa tonicité posturale, ni dans les échanges de regards.

La tristesse d'une rencontre qui ne prend pas et qui ne trouve pas son authenticité avait rendu peu expressives la mère comme le bébé. Pour arriver à faire face, cette installation en faux-self de la maman, qui voulait pourtant 
bien faire malgré les difficultés rencontrées avec sa fille, ne pouvait pas confirmer à la petite le ressenti déprimant de la relation. Aujourd'hui, l'impassibilité du visage de Flora traduit encore le peu d'expressions qu'elle a reçu du visage premier maternel et de sa difficulté d'accordage.

La sécurité d'un détour ou de l'éprouvé d'un fond ou de bords solides dans l'environnement sont rassurants comme l'est la prévisibilité des mouvements de l'environnement.

\section{AUTHENTICITÉ DES ÉMOTIONS EXPRIMÉES}

Quand je rencontre Flora, alors qu'elle a une dizaine d'années, je suis surprise de la voir à certains moments déployer une force incroyable comme dans une nécessité absolue de se sentir exister. Son corps semble se maintenir par une carapace musculaire extrême qui lui crée une posture et une démarche très enraidies et peu ajustées.

Pendant une période, Flora a l'art d'attraper mes grands cheveux juste avant d'entrer dans mon bureau, pour s'y suspendre avec toute sa force, en me fixant avec un visage impassible, les yeux rivés sur mon visage en attendant une réaction. J'évite dans un premier temps qu'elle ne m'arrache effectivement des poignées de cheveux en les retenant à la racine. Très vite, ma réaction d'essayer de faire face avec bienveillance ne suffit pas à désamorcer ses manœuvres. Cela semble au contraire les susciter ou les renforcer.

Je suis étonnée qu'en tirant mes cheveux avec toute sa force, elle focalise son regard sur mon visage, tandis que le sien est totalement inexpressif. L'appel à la raison, à la morale ou à la loi interdisant de faire mal aux autres, ou des tentatives d'explications que cela me fait trop mal ne sont d'aucune efficacité. J'en viens à me demander si elle-même, qui a les cheveux longs, a eu l'expérience de la douleur pendant le coiffage du matin et si elle a senti qu'on lui tirait les cheveux, comme je pouvais le ressentir. Je reste sans réponse.

Je ne la sens pourtant pas dans une intention violente à mon égard et je ne ressens pas chez elle de décharge d'agressivité. Elle semble pourtant déterminée dans son geste avant d'entrer dans le bureau à attendre une réponse précise que je ne peux visiblement pas encore lui apporter. Je me trouve alors un peu trop bienveillante à son égard, à mon détriment, ce qui me fait prendre conscience du contre-transfert dans lequel je suis installée.

Comme sa mère, je veux bien faire, et je me vois installée en faux-self. Probablement sa mère a dû longtemps vouloir le meilleur pour sa fille sans être connectée à ses ressentis ni à ses émotions personnelles dans la rencontre. Cette prise de conscience me met davantage en contact avec une 
réaction naturelle face à la douleur que nous pouvons avoir quand quelqu'un nous tire les cheveux brusquement avec force.

J'emploie alors le langage des émotions en lui disant avec emphase que si elle continue à me tirer les cheveux, je vais pleurer, parce que cela me fait très mal, tout en mimant sur mon visage la douleur et un début de pleurs. Cette réponse, cette fois-ci enfin authentique, la satisfait au point qu'elle lâche immédiatement mes cheveux pour me donner gentiment la main en m'entraînant dans le bureau. Visiblement Flora veut réveiller ce visage maternel peu expressif à travers le mien pour que l'expression de visage soit en lien avec les émotions et les ressentis éprouvés, avant d'entrer en séance.

L'authenticité de la relation thérapeutique est une des conditions à une rencontre possible. Elle permet de transformer des installations en faux-self en une adéquation entre le vécu émotionnel interne et son expression.

\section{LA RELATION « CASSÉE »}

Flora est encore sans langage verbal. Sa bouche est très peu investie. Elle a tendance à réanimer sa bouche par des sensations qu'elle se procure, par une stéréotypie qui consiste à se lécher rapidement l'intérieur de ses mains pour mouiller ensuite d'un geste bref dans un mouvement symétrique le pourtour de sa bouche, de haut en bas, jusqu'à se provoquer une irritation dermique permanente. Les somatisations ou les automutilations sur les zones corporelles en cours d'investissement sont fréquentes chez les enfants autistes. Faut-il encore qu'un autre puisse partager par le langage cette tentative d'investissement pour que les stéréotypies s'estompent et que l'investissement sensoriel renouvelé à chaque instant s'inscrive dans la durée. Cette réanimation permanente de la zone buccale laisse présager une potentialité d'accès au langage verbal chez Flora.

Avec le bénéfice de la psychothérapie, elle commence en effet à émettre quelques sons, chuchotés dans un premier temps, puis prononce un mot à peine audible que je finis par décoder : « cassé ». Elle semble avoir déplacé son intérêt pour la relation dans des métaphores de la relation prises dans l'environnement non humain qu'elle commence à partager avec moi dans une attention conjointe. Tout ce qui est « cassé » devient une véritable énigme pour elle. Elle fait son enquête en observant minutieusement les objets ou les feuilles « cassées » qu'elle déchire préalablement dans un angle, comme si cela lui permettait, au fond, de s'interroger sur l'énigme d'un échange ou d'un regard « cassé ».

Flora se comporte avec les objets de telle sorte qu'elle vérifie si elle peut avoir un impact sur eux et si elle peut les faire réagir ou les animer. Elle parle aux feuilles cassées dans un jargon incompréhensible en chuchotant 
pour elle-même et dans une grande attention. Elle perd alors la notion de la différence entre ce qui est humain et non humain. Non pas inhumain, car elle essaie au contraire d'humaniser des objets inertes, sous son contrôle, comme pour déclencher une transformation du non humain en humain. Par sa persévérance, dans une pensée magique, elle paraît convaincue qu'elle pourrait y parvenir.

Nous savons combien il est difficile pour un enfant autiste d'investir le regard en direct avec un proche. Flora se passionne pourtant pour l'observation des objets environnementaux, à partir du moment où ils ne sont pas humains. Le choix de ces objets pris dans l'environnement fait étrangement résonance avec une attirance pour des qualités relationnelles et des composantes sensorielles nécessaires à l'investissement de l'image du corps ou de la relation. Ces objets en seraient des métaphores.

Tant que la relation en direct est évitée, l'objet environnemental non humain en devient un équivalent encore peu différencié. Le choix des objets environnementaux est pris dans le lien métaphorique de la relation ou de l'image du corps en devenir quant aux qualités sensorielles ou émotionnelles recherchées.

\section{LA MISE EN MOUVEMENT DANS LA PENSÉE MAGIQUE}

Un jour où elle se sent en confiance dans le cadre de nos rencontres, elle se met à grimper sur mon bureau en mobilisant toute sa force et s'installe tranquillement, assise en tailleur, face à moi, avec l'assurance qu'elle peut occuper cet espace de droit. J'ai l'impression d'avoir sous les yeux, sur mon bureau, un grand bébé de 10 ans assis à hauteur de mes bras, prêt à tenter l'expérience des échanges de regards avec moi. Mais Flora semble au préalable s'assurer d'une certaine sécurité avant de se lancer dans cette aventure.

Sur sa droite, du haut de son perchoir, elle aperçoit un ballon dans le bas du placard. Elle lui fait un clin d'œil séducteur accompagné d'un geste de son index comme pour le convier à la rejoindre de façon complice. J'ai alors l'impression que sa pensée d'obtenir le déplacement du ballon lui donne l'assurance que celui-ci pourrait venir de façon magique. Je mesure combien les humains et les objets non humains ont été, pour elle, interchangés. J'ai le sentiment qu'il est plus simple pour elle que je sois reléguée à occuper la place d'un objet inerte et d'investir le ballon de façon vivante comme s'il était un interlocuteur privilégié plus contrôlable. Aucune manifestation d'échange en direct n'est encore adressée dans ma direction. Seule sa présence massive sous mes yeux me rend à l'évidence que tout cela m'est adressé malgré tout. 
Le lien aux objets environnementaux peut se faire dans une pensée magique, particulièrement quand il s'agit du déplacement des objets non humains qui serait attendu de façon prévisible là où l'imprévisibilité des mouvements humains serait difficilement supportable.

Dans le cadre thérapeutique avec Flora, le passage par l'illusion que la pensée suffirait à faire déplacer des objets environnementaux a été un préalable à une confiance retrouvée dans le lien qui a pu s'établir en direct par la suite.

Mes commentaires visent à raconter ces beaux échanges qu'elle voudrait bien avoir avec le ballon. C'est ainsi que ce qu'elle met en scène dans ces échanges complices avec le ballon devient partageable entre elle et moi. Si Flora n'est pas encore capable d'exprimer une demande à mon égard, elle peut tout de même profiter de mes commentaires.

Je sens que l'illusion que le ballon pourrait venir par magie en le regardant «droit dans les yeux » et en l'appelant d'un clin d'œil puis d'un signe avec son index pourrait l'aider à se préparer à avoir des échanges en direct par la suite. C'est pourquoi je vais chercher le ballon pour répondre à son attente en le lui donnant. Elle le saisit immédiatement. Puis je me réinstalle sur ma chaise, à ma place, en face d'elle qui est toujours perchée en tailleur sur mon bureau sous mes yeux.

Elle commence par attaquer le rideau en projetant le ballon sur cette forme inquiétante. Je me relève pour lui restituer le ballon tout en commentant à la cantonade qu'il est parfois difficile de regarder des endroits inquiétants qui peuvent changer de forme comme le rideau ou mes yeux, et qu'en y lançant le ballon, cela permet de se préparer à regarder. Puis je la rassure en lui disant que lorsqu' on se regarde, bien que les yeux et le visage bougent un peu, le fond du regard est toujours bien solide.

À d'autres moments, Flora lance des objets vers la direction du fond sombre inaccessible du haut d'un placard qui paraît lui faire aussi peur que les rideaux ou mon regard. J'ai le sentiment qu'elle me montre sa crainte de se lancer dans mon regard de peur de s'y perdre. Je lui restitue le ballon afin qu'elle fasse l'expérience qu'un ballon ou qu'un regard lancé ne se perd pas dans un trou noir sans fond.

Après ces commentaires interprétatifs et mon déplacement pour rechercher le ballon et le lui restituer, je vois Flora se saisir du ballon, toujours assise en tailleur sur mon bureau. Dès qu' elle a repris le ballon, elle le met en contact doucement sur sa tête puis sur les bords de son corps, comme pour éprouver la contenance et l'intégrité de son enveloppe corporelle avant tout échange relationnel. Elle manifeste à plusieurs reprises que le ballon ne doit pas quitter l'espace du bureau comme pour garantir une limite environnementale à son propre corps et pour contrôler son existence corpo- 
relle. Je veille à ce que le ballon ne tombe pas du bureau. Je confirme par mes commentaires son intégrité corporelle. Elle touche les bords de la table comme pour éprouver un espace de sécurité.

Elle réussit alors spontanément à me lancer le ballon tout en m'adressant un regard. Puis elle réclame le retour du ballon comme pour s'assurer qu'elle n'est pas tombée dans mon regard, que je ne vais pas l'absorber et qu'elle ne disparaît pas si elle investit la relation avec moi. Nous savourons nos premiers échanges de regards en direct qu'elle a préparés en traçant dans l'espace ces allers et retours avec le mouvement du ballon, d'abord dans l'environnement non humain vers le rideau puis vers le fond du placard et entre nous deux.

La difficulté de Flora, comme d'autres enfants autistes, à percevoir les mouvements et les déplacements dans l'espace l'a conduite à s'aménager dans une pensée magique : le mouvement du ballon allait sans doute se faire tout seul par son invitation à distance et par l'interchangeabilité des registres humains et non humains. Ce passage par une pensée magique à laquelle j'ai répondu en lui faisant obtenir le ballon qu'elle voulait a permis par la suite qu'elle retrouve spontanément un échange plus direct en face à face. Je pense que cette pensée magique a contourné les difficultés de perception des mouvements dans l'espace qui sont plus intégrables s'ils sont contrôlés ou s'ils sont ralentis. C'était en la voyant tourner sa tête très lentement dans ma direction devant le massif de fleurs que je comprenais l'importance pour elle des déplacements au ralenti afin qu'ils soient intégrables.

\section{INDIFFÉRENCIATION ENTRE HUMAIN ET NON HUMAIN ?}

Cette interchangeabilité de la relation entre le registre humain et le registre non humain traduit-elle une indifférenciation entre humain et non humain ? Chez Flora, comme chez d'autres enfants autistes, je ne pense pas qu'il y ait une indifférenciation entre humain et non humain mais plutôt un évitement de la relation en direct avec les autres, au profit d'un surinvestissement de la relation avec l'environnement non humain plus contrôlable, dans une visée de diminuer la charge émotionnelle provoquée par l'angoisse de l'imprévisibilité de l'accordage des regards. Prendre le risque que l'accordage ne se fasse pas à son rythme équivaudrait-il à un risque de chute sans fin et sans fond d'un regard non contenant? Probablement. De plus, la difficulté à évaluer la profondeur de l'espace et les mouvements dans l'espace lui fait préférer les mouvements non humains plus prévisibles dans leurs déplacements.

Cet évitement de la rencontre en direct n'exclut pas pour autant une rencontre par la suite. Car l'expérience de la rencontre avec l'environnement 
non humain, par exemple avec le fond du placard, le rideau, la table ou par le biais du ballon est pour Flora comme un passage obligé pour interroger avec une intensité émotionnelle moindre, la capacité contenante de l'environnement en général. Ce sont les mêmes processus psychiques de déplacement et de recours au détour métaphorique à l'œuvre dans la formation des rêves qui apaisent l'intensité trop forte de contenus encore trop chargés pour qu'ils puissent s'exprimer directement.

Tant que l'environnement non humain n'est pas partageable avec un autre, aucune contenance humaine ne peut y être associée. C'est par la présence d'un autre et par ses commentaires qui donnent du sens à ses manœuvres que l'évitement préalable d'une rencontre en direct devient spontanément réversible pour Flora.

Ainsi lorsque les recherches sensorielles des bords, du fond et de la contenance de l'environnement non humain deviennent partageables, elles peuvent déclencher par résonance un intérêt spontané pour la relation en direct afin d'obtenir des sensations similaires.

\section{PENSÉE ET ACTION MAGIQUES}

La pensée magique aurait-elle un lien avec le trouver-créer de Winnicott, l'illusion que le bébé éprouve de créer le bon sein apportant le lait quand il obtient immédiatement satisfaction ? Il suffirait de penser à une action pour qu'elle se réalise, dans une pensée toute-puissante qui apporterait satisfaction ou apaisement.

Flora veut obtenir le ballon. Il suffit pour elle qu'elle y pense et qu'elle l'amadoue d'un clin d'œil accompagné d'un signe d'invitation avec sa main pour qu'il vienne de lui-même.

La magie aurait-elle un lien avec le monde inanimé non humain au point que celui-ci s'anime spontanément et qu'il devienne humain ? Le petit d'homme arrive pourtant normalement équipé de la capacité à distinguer et à préférer le mouvement lorsqu'il est humain, celui des yeux en particulier sur le visage maternel, ce qui fait partie des mouvements biologiques. Il délaisse alors les objets non humains inanimés, à moins qu'ils ne commencent à s'animer comme le font les objets mécaniques par exemple.

Il peut normalement associer la mise en mouvement naturelle d'une personne ou d'un animal au registre du vivant. C'est-à-dire qu'il trouve normal qu'une personne se mette en mouvement spontanément sans qu'elle ait été bousculée par un autre corps en mouvement. Par contre il trouve anormal qu'un objet non humain se mette spontanément en mouvement à moins d'avoir été heurté par une force en mouvement d'un autre corps. C'est pourquoi les objets non humains inanimés qui s'animent ou les personnes 
inanimées qui restent immuables paraissent toujours étranges. Freud en parlait déjà à propos d'expériences d'inquiétante étrangeté ${ }^{3}$.

Il n'est pas rare de voir qu'une personne au visage inanimé, peu expressive, suscite davantage d'agressivité au visage qu'une personne expressive. L'inexpressivité d'un visage provoque une grande inquiétude comme s'il n'appartenait plus au monde humain ou animal, par son incapacité à bouger spontanément. Il faudrait désormais le bousculer comme un objet non humain pour qu'elle s'anime. Les personnes dépressives ou des personnes installées en faux-self suscitent cette inquiétude car elles s'animent peu ou de façon peu authentique et parfois mécanique. Traiter des personnes comme des objets ou considérer des objets comme des personnes deviendrait alors nécessaire et relèverait alors d'un passage par une pensée magique, le temps de retrouver des repères tangibles de différenciation entre ces deux registres.

Flora n'aurait ainsi pas pu différencier assez tôt les mouvements non humains des mouvements humains tant que l'accordage ne se faisait pas entre elle et sa mère. L'expérience du bébé d'être imité ou d'être commenté de façon spontanée par sa mère dans un accordage émotionnel lui permet d'avoir l'expérience que les humains réagissent sur leur visage ou dans leur motricité de façon spontanée sans qu'ils aient besoin d'être heurtés par l'impact d'un contact en direct qui provoque un mouvement. Ce sont les émotions que la mère éprouve pour son bébé qui la mobilisent, qui provoquent une première mise en mouvement pour qu'elle s'anime et anime son bébé. Elle est aussi un miroir identifiant et vivant pour son enfant. Winnicott nous a sensibilisés à l'importance de la " préoccupation maternelle primaire ". Lorsque des troubles de l'intégration sensorielle imposent au bébé ou à un enfant autiste un rythme plus lent ou lorsque la mère est déprimée ou trop soucieuse, l'accordage ne peut se faire aisément. Ce décalage émotionnel ou de réactions fait perdre ainsi la possibilité de différencier le registre humain de celui qui est non humain.

Le recours à la pensée magique qui donne l'illusion que la mise en mouvement d'un objet inerte serait interchangeable avec celui d'un humain permettrait de conserver cet état d'indifférenciation qui protège provisoirement de la détresse de ne pas se sentir exister dans le miroir d'un autre. Si le thérapeute accepte de dialoguer avec un enfant autiste dans cette interchangeabilité des registres humains et non humains dans un premier temps, un partage émotionnel reste possible et l'enfant peut alors faire l'expérience sécurisée de la rencontre sur un plan plus large. Il peut se sentir alors exister

3. S. Freud (1919), "L'inquiétante étrangeté », dans Essais de psychanalyse appliquée, Paris, Gallimard, coll. "Idées », 1933, réimpression 1971, NRF, n² 263, p. 163-210. 
dans le lien à un autre et il réinvestit peu à peu le lien en direct de lui-même et à son rythme.

L'expérience $d u$ ballon et du regard lancés puis récupérés dans un dialogue gestuel qui s'est installé avec Flora, accompagnée de mes commentaires, lui a donné la confiance requise pour tenter de me faire confiance dans tout son être, tel un petit enfant qui se lance entièrement dans les bras de l'un de ses parents, en se jetant littéralement dans l'espace pour se laisser choir, avec la conviction qu'il sera accueilli, retenu et contenu sans perdre sa forme ni son sentiment d'existence.

Flora en fait spontanément l'expérience quelques mois après le début de la thérapie alors que je la raccompagne dans son groupe. Elle prenait depuis peu l'habitude de me suivre pendant le trajet sans plus me donner la main, mais en se tenant à distance dans un accordage à mes déambulations, comme pour commencer à se différencier en ma présence.

Ce jour-là, Flora est perchée sur le talus qui surplombe à gauche le chemin que j'emprunte. Ce chemin est bordé par une barrière à ma droite. Sa démarche est encore enraidie par son installation en carapace musculaire. Elle commence pourtant, à cette époque, à se lancer de plus en plus souvent de façon fugitive dans mon regard.

Je sens Flora joyeuse et confiante dans la relation. Après un coup d'œil furtif qui tend à s'installer dans ma direction, elle se lance vers moi dans tout son corps, bras en avant et se jette avec toute sa force du haut du talus, le corps encore maladroit et à peine coordonné. Je l'accueille en ouvrant mes bras comme j'aurais pu le faire avec un tout petit qui fait l'expérience de son premier saut du haut d'un muret. Je réceptionne Flora avec la force de son élan et le poids de ses 13 ans, grâce au renfort d'arrière-plan de la barrière qui se trouve dans mon dos. Elle peut ainsi faire l'expérience avec confiance d'un fond solide qui la retient, sans évaluer pour autant l'inappropriation du geste d'un tout-petit dans un corps de grande fille. Je suis touchée par la beauté de ce geste de confiance et je le lui témoigne. Elle-même en paraît tout émue également. Elle commence à éprouver du plaisir à nos échanges. L'attention conjointe sur les feuilles « cassées » qui la fascinaient nous avait déjà rendu grand service pour partager et nous émerveiller à deux sur ses intérêts.

C'est dans cette période qu'elle me témoigne également toute sa confiance quand elle commence à s'intéresser à la pâte à modeler que je mets à sa disposition. Pendant quelques séances elle en ôte un petit morceau, comme elle avait pu casser un petit morceau aux feuilles devenues " cassées ». Dans un premier temps elle jette n'importe où ces petits bouts détachés qui se trouvent ainsi dispersés dans la pièce. J'ai l'impression que tant qu'elle est elle-même dispersée, elle ne peut pas se rassembler sans la relation à l'autre. Mais ce jour-là, après avoir détaché un tout petit bout de 
pâte à modeler, elle l'enfourne en un instant dans ma manche comme pour me confier sa partie cassée afin que je puisse la protéger ou la réparer dans la relation transférentielle. J'ai l'impression qu'elle vient se loger dans le creux de mes bras en peau à peau, de façon magique par le biais de ce bout de pâte à modeler.

La recherche des qualités échangées normalement dans la relation avec un autre primordial s'est déplacée vers les formes de l'environnement non humain, sans la conscience de ces déplacements. C'est ainsi que Flora attend la réponse donnée par l'environnement non humain là où elle n'attend plus de réponse du côté humain.

C'est ainsi qu'elle semble interroger la capacité contenante de la flaque d'eau en testant le fond avec son pied comme pour indiquer son attente de contenance de l'environnement que je représente dans le transfert dans les débuts de séances.

Quand elle attend du ballon qu'il vienne sans qu'elle se déplace pour aller le chercher, sans penser me solliciter non plus, et sans que le ballon reçoive un impact, elle fait l'économie du langage verbal adressé dans la relation en direct avec un autre. Sa pensée magique et son langage non verbal y participent et tentent un accordage immédiat pour obtenir satisfaction en interchangeant les registres humains et non humains.

Puis elle condense des moments de sa petite enfance avec le moment transférentiel où elle cherche à provoquer des réactions émotionnelles qui feraient réagir sa mère et moi-même prises dans des moments d'installation en faux-self pour mieux profiter d'une relation en direct.

Enfin elle peut se lancer directement dans mes bras même maladroitement avec son corps peu ajusté ni très souple.

La pensée magique qui tendrait à faire oublier les déplacements d'investissement entre le monde humain et non humain permettrait de ne pas perdre le contact avec les qualités premières recherchées dans la relation à l'autre. Le détour métaphorique de la relation par un environnement non humain inanimé, en attente de réanimation, pourrait être considéré comme un passage nécessaire qui prépare à la relation en direct. Faut-il encore qu'un autre s'intéresse à ce passage par la métaphore pour créer une rencontre première.

Ainsi la pensée magique ferait l'économie de la pensée logique et de l'adéquation avec le contexte de la situation pour ne pas perdre le fil conduc- 
teur du lien à l'autre, dans sa potentialité relationnelle, que l'objet soit provisoirement humain ou non humain.

Le langage gestuel adressé avec complicité au ballon n'est pas pour autant délirant. Il n'utilise pas le processus de la projection que l'on trouverait dans un registre psychotique, mais il vise simplement à faire l'expérience sensorielle, motrice et émotionnelle du lien à l'autre, ce grand autre environnement dans un sens large au-delà de l'humain.

Tel un bébé qui n'a pas encore acquis l'usage de sa motricité dans ses propres déplacements pour obtenir ce dont il a besoin, Flora, qui n'a pu encore intégrer la fonction contenante de l'environnement humain, userait de sa pensée magique pour obtenir ce dont elle a besoin pour se sentir exister avec confiance. C'est par le détour d'expériences d'indifférenciation entre la mise en mouvement d'objets non humains comme le ballon, dans l'illusion qu'elle a provoqué son arrivée, et dans l'expérience magique de condamner provisoirement le thérapeute à l'expérience de l'inanimé, qu'elle accédera ensuite dans le meilleur des cas à son individuation et à la relation en direct.

Quand le thérapeute peut devenir conscient de la valeur métaphorique du lien au monde humain que l'enfant autiste entretient avec les objets non humains environnementaux ou avec le monde végétal, il devient le garant d'une rencontre possible, au rythme de l'enfant.

C'est le jeu partagé qu'offre le thérapeute avec la pensée magique de l'enfant qui permet à celui-ci de trouver et créer petit à petit la confiance de se lancer dans la relation en direct avec un semblable, tout en conservant l'assurance que l'environnement non humain reste potentiellement un recours dans les moments difficiles, car il est plus prévisible.

C'est sans doute dans un état de résonance immédiat avec ses constructions internes en devenir que l'enfant négocie avec l'environnement non humain dans une pensée magique. Cette pensée magique prend valeur de métaphore d'un accordage immédiat. L'enfant autiste peut ainsi s'appuyer provisoirement et compter sur cet environnement non humain prévisible pour accéder à son rythme à la relation en direct avec les humains, mais à condition qu'un autre, un thérapeute, y prête attention et considère le jeu avec la pensée magique de l'enfant comme une étape structurante.

\section{BIBLIOGRAPHIE}

DESJARDINS, V. ; GOLSE, B. 2004. « Du corps, des formes, des mouvements et du rythme comme précurseurs de l'émergence de l'intersubjectivité et de la parole chez le bébé », Journal de la psychanalyse des enfants, $\mathrm{n}^{\circ} 35$, "Le langage ", Paris, Bayard, p. 171-191. 
FrEUD, S. 1919. "L'inquiétante étrangeté », Essais de psychanalyse appliquée, Paris, Gallimard, coll. «Idées », 1933, réimpression 1971, NRF, n² 263, p. 163-210.

GEPNER, B. 2006. «Constellation autistique, mouvement, temps et pensée. Malvoyance de l'É-Motion, autres désordres du traitement temporospatial des flux sensoriels et dyssynchronie dans l'autisme ", Devenir, vol. 18, n 4, Chêne-Bourg, Éditions Médecine \& Hygiène, p. 333-379.

LheureuX-DAVIDSE, C. 2003. L'autisme infantile ou le bruit de la rencontre. Contribution à une clinique des processus thérapeutiques, Paris, L'Harmattan, 342 p.

LHEUREUX-DAVIDSE, C. 2004. « La reconstruction de l'image du corps chez Léo, enfant trisomique présentant des retraits autistiques », Cliniques méditerranéennes, $\mathrm{n}^{\circ} 69$, Toulouse, érès, p. 289-308.

LHEUREUX-DAVIDSE, C. 2007. "Jouer avec les mouvements, les vibrations et les rythmes dans l'émergence de la voix », Champ psychosomatique, $\mathrm{n}^{\circ} 48$, « La voix », Paris, L'Esprit du temps, p. 185-203.

Meltzer, D. 1975. Explorations in Autism, Strath Tay, Clunie Press, trad. Explorations dans le monde de l'autisme, Paris, Payot, 1980, 266 p.

Meltzer, D. ; Williams, M. H. 1988. The Apprehension of Beauty. The Role of Aesthetic Conflict in Development, Violence and Art, Strath Tay, Clunie Press, trad. D. Alcorn, préf. D. Houzel, L'appréhension de la beauté. Le conflit esthétique, son rôle dans le développement, la violence, l'art, Larmor-Plage, Éditions du Hublot, 2000, 254 p.

STERN, D. 2003. Le monde interpersonnel du nourrisson, Paris, PUF, coll. « Le fil rouge ». Williams, D. 1992. Nobody Nowhere, trad. Si on me touche, je n'existe plus, Le récit d'une autiste, Paris, Robert Laffont, coll. «Vécu ».

WinnicotT, D. W. 1971. Playing and Reality, trad. C. Monod et J.-B. Pontalis (1975), Jeu et réalité, l'espace potentiel, Paris, Gallimard, 1997, 212 p.

\section{Résumé}

La rencontre en direct pour un enfant autiste est souvent très difficile au point que le contact peut entraîner chez lui le sentiment de disparaître. Par des installations en clivage et de dispersion psychique, l'enfant est davantage dans une pensée associative qui risque de dériver que dans une pensée logique et contextualisée. Dans le langage verbal, lorsqu'il émerge, l'environnement associatif du mot désigné peut être verbalisé avant que le mot lui-même puisse être prononcé. La sécurité d'un détour par l'environnement non humain et l'éprouvé d'un fond ou de bords solides de l'espace sont rassurants par leur prévisibilité et leur stabilité, avant qu'un investissement des mouvements dans l'espace ou dans la relation puisse être tolérable et intégrable. L'authenticité de la relation thérapeutique favorise la transformation d'une installation en faux-self en une adéquation entre un vécu émotionnel interne et son expression. Elle permet d'appréhender la perception des changements émotionnels sur un fond de stabilité plus rassurant. Mais l'objet environnemental non humain devient privilégié chez l'enfant autiste et interchangé avec le monde environnant humain jusqu'à des expériences d'indifférenciation.

Le lien aux objets environnementaux non humains peut s'établir selon une pensée magique, particulièrement quand il s'agit de leur déplacement et de leurs mouve- 
ments. Les personnes autistes tentent de les maîtriser au moment où les mouvements humains trop imprévisibles restent encore ingérables. L'investissement du lien lorsqu'il est construit avec confiance, sécurité et de façon prévisible est la condition d'un meilleur investissement de l'espace, des mouvements et des déplacements.

Dans un cadre thérapeutique, le passage par une expérience d'illusion que les objets inanimés peuvent se déplacer par une pensée magique peut être un préalable à une confiance retrouvée dans le lien en direct. La pensée magique concerne particulièrement les mises en mouvement des objets inanimés pour mieux conserver la potentialité d'un accordage relationnel. Si le thérapeute accepte de dialoguer avec un enfant autiste dans cette interchangeabilité des registres humains et non humains dans un premier temps, un partage émotionnel reste possible. Le jeu avec la pensée magique de l'enfant y contribue et il réinvestit plus souvent et spontanément le lien en direct.

Mots-clés

Autisme, espace, environnement, mouvement, pensée magique.

BRINGING INTO MOVEMENT IN MAGICAL THOUGHT

Summary

Meeting directly is often very difficult for an autistic child, to such a point that contact can give him the feeling of disappearance. By splitting and psychic dispersal, the child is in an associative mode of thinking, which risks to divert, rather than thinking logically and within context. In the verbal language, when this emerges, the associative environment of the indicated word can be put into words before the word itself can be pronounced. The safety of a detour by the non human environment and experiencing the floor or the solid edges of a space are reassuring by their previsibility and their stability, before an investment of movements in space or in the relation can be bearable and integrable.

The authenticity of the therapeutic relationship facilitates the transformation of an installation in the false self into an adequacy between the internal emotional and its expression. The therapeutic relationship permits to apprehend the perception of changing emotions on a firmer, more reassuring basis. But the non human object in the environment remains preferred by the autistic child, and interchanged with the human surrounding world until it has experiences of indifferentiation.

The link with the non human objects in the environment may in particular show magical thinking when it concerns their movements and relocations. Autistic persons tend to master them when human movements are too impredictable and still remain unmanageable. The investment of the link, when it is built with confidence, security and in a predictable way, is the condition of a better investment of the space, movements and the relocations.

In a therapeutic setting passing through an illusory experience that inanimated objects can relocate themselves by a magical thought, may be preliminary to a re-found confidence in meeting directly. Magical thoughts concern in particular 
bringing inanimate objects into movement to better conserve the potentiality of attunement in relationship. If the therapist at first accepts to dialogue with an autistic child within this interchangeability of human and non human registers, an emotional sharing remains possible. The play with the magical thoughts of the child contributes and reinvests often spontaneously meeting directly.

Keywords

Autism, space, environment, movement, magical thought. 